\title{
POSITIVE AND RE-POSITIVE SOLUTIONS TO SOME SYSTEMS OF ADJOINTABLE OPERATOR EQUATIONS OVER HILBERT $C^{*}$ - MODULES*
}

\author{
GUANG JING SONG ${ }^{\dagger}$ AND QING WEN WANG ${ }^{\ddagger}$
}

Abstract. A necessary and sufficient condition for the existence of the general common positive solution to equations

$$
A_{1} X=C_{1}, \quad X B_{2}=C_{2}, \quad A_{3} X A_{3}^{*}=C_{3}, \quad A_{4} X A_{4}^{*}=C_{4}
$$

for operators between Hilbert $C^{*}$-modules is established, and an expression for the common positive solution to the equations is derived when the solvability conditions are satisfied. As an application, a new necessary and sufficient condition for the system of adjointable operator equations

$$
A X=C, X B=D
$$

over Hilbert $C^{*}$-modules to have a common Re-positive solution is proved. Moreover, an expression of the general Re-positive solution is derived when the consistent conditions are met. The results of this paper extend some known results in the literature.

Key words. Hilbert $C^{*}$-module, Moore-Penrose inverse, Operator equation, Positive solution, Re-positive solution.

AMS subject classifications. 47A62, 47B15, 47B65, 15A09, 15A24.

1. Introduction. Hermitian positive semidefinite solutions to some matrix equations or some operator equations were investigated by many authors. For finite matrices, Khatri and Mitra [12] gave necessary and sufficient conditions for the existence of a common positive semidefinite solution to equations

$$
A X=C, \quad X B=D
$$

over the complex field $\mathbb{C}$, and presented an explicit expression for the general Hermitian positive semidefinite solutions to system (1.1) by generalized inverses, when the

${ }^{*}$ Received by the editors on February 23, 2011. Accepted for publication on October 4, 2011. Handling Editor: Michael Tsatsomeros.

${ }^{\dagger}$ School of Mathematics and Information Sciences, Weifang University Weifang 261061, P.R. China (sgjshu@yahoo.com.cn). Supported by grants from Natural Science Foundation of Shan Dong Province (no. ZR2010AL107) and the Ph.D. Foundation of Weifang University (no. 2011BS12).

${ }^{\ddagger}$ Department of Mathematics, Shanghai University, Shanghai 200444, P.R. China (wqw858@yahoo.com.cn). Supported by grants from the National Natural Science Foundation of China (no. 11171205), the Natural Science Foundation of Shanghai (no. 11ZR1412500), and the Ph.D. Programs Foundation of Ministry of Education of China (no. 20093108110001). 
solvability conditions were satisfied. Baksalary [1] and Groß [9] studied the nonnegative definite and positive definite solutions to matrix equation

$$
A X A^{*}=B
$$

respectively. In 2007, Cvetković-Ilić et al. [2] investigated the positive solution to equation (1.2) in $C^{*}$-algebras. As a generation of equation (1.2), Zhang [26] derived a expression of the general nonnegative definite solution to system of matrix equations

$$
A_{3} X A_{3}^{*}=C_{3}, \quad A_{4} X A_{4}^{*}=C_{4}
$$

over the complex field $\mathbb{C}$. Some other results can be found in $[4-6,15,16,19,24,25]$.

Meanwhile, the Re-positive solution to some matrix equations or some operator equations is also active. Wu [22] studied Re-positive solutions of

$$
A X=C .
$$

$\mathrm{Wu}$ and Cain [23] found the set of all complex Re-nnd (Re-nonnegative definite) matrices $X$ satisfied

$$
X B=D
$$

and presented a criterion for Re-nndness. Groß [8] gave an alternative approach, which simultaneously delivers explicit Re-nnd solutions and gave a corrected version of some results from [23]. Beside these papers, many other papers have dealt with the problem of finding the Re-nnd and Re-pd (Re-positive definite) solutions of some other forms of equations (see e.g. [3,20]). Dajić and Koliha [5] reviewed system (1.1) from a new perspective by studying them in the setting of associative rings with or without involution and derived a general form of Re-positive solutions of equation (1.4) over the Hilbert spaces. Unfortunately, the investigation of the common positive solutions cannot be applied to the common Re-positive solutions to system (1.1), since that method does not work for solutions which are not necessarily Hermitian.

Recently, Wang and Wu [18] presented an expression for general Hermitian solution to system of equations

$$
A_{1} X=C_{1}, X B_{1}=C_{2}, A_{3} X A_{3}^{*}=C_{3}, A_{4} X A_{4}^{*}=C_{4}
$$

over Hilbert $C^{*}$-modules. To our knowledge, so far there has been little information on either the common positive solution to (1.5) for operators in the framework of Hilbert $C^{*}$-modules, or the Re-positive solution to system (1.1) of matrix equations over the complex field and adjointable operator equations over Hilbert $C^{*}$-modules.

Motivated by the work mentioned above, we in this paper aim to consider the necessary and sufficient conditions for a system of adjointable operator equations (1.5) 
(or system (1.1)) to have a positive solution (Re-positive solution), as well as present an expression for the general positive solutions (Re-positive solutions) to this system when the consistent conditions are satisfied.

The paper is organized as follows. In Section 2, we begin with some basic concepts and results about adjointable operators and generalized inverse of adjointable operators over Hilbert $C^{*}$-modules. In Section 3, we give a necessary and sufficient condition for the existence of a positive solution to system (1.5) of adjointable operator equations over Hilbert $C^{*}$-modules. When the solvability conditions are met, we present an expression for the general positive solution to system (1.1). As an application, in Section 4, we show a necessary and sufficient condition for system (1.1) of adjointable operator equations over Hilbert $C^{*}$-modules to have a common Repositive solution, and derive an expression of the general Re-positive solution when the consistent conditions are met. To conclude this paper, in Section 5, we propose some further research topics.

2. Preliminaries. Hilbert $C^{*}$-modules arose as generalizations of the notion of Hilbert space. The basic idea was to consider modules over $C^{*}$-algebras instead of linear spaces and to allow the inner product to take values in a $C^{*}$-algebra. The structure was first used by Kaplansky [11] in 1952. For more details of $C^{*}$-algebra and Hilbert $C^{*}$-modules, we refer the readers to [13] and [21].

Let $\mathfrak{A}$ be a $C^{*}$-algebra. An inner-product $\mathfrak{A}$-module is a linear space $E$ which is a right $\mathfrak{A}$-module (with a scalar multiplication satisfying $\lambda(x a)=x(\lambda a)=(\lambda x) a$ for $x \in E, a \in \mathfrak{A}, \lambda \in \mathbb{C})$, together with a map $E \times E \rightarrow \mathfrak{A},(x, y) \rightarrow\langle x, y\rangle$ such that

(1) $\langle x, \alpha y+\beta z\rangle=\alpha\langle x, y\rangle+\beta\langle x, z\rangle$;

(2) $\langle x, y a\rangle=\langle x, y\rangle a$;

(3) $\langle x, y\rangle=\langle y, x\rangle^{*}$

(4) $\langle x, x\rangle \geq 0$, and $\langle x, x\rangle=0 \Leftrightarrow x=0$

for any $x, y, z \in E, \alpha, \beta \in \mathbb{C}$ and $a \in \mathfrak{A}$. An inner-product $\mathfrak{A}$-module $E$ is called a (right) Hilbert $\mathfrak{A}$-module if it is complete with respect to the induced norm $\|x\|=$ $|\langle x, x\rangle|^{1 / 2}$.

Assume that $\mathcal{H}$ and $\mathcal{K}$ are two Hilbert $\mathfrak{A}$-modules, and $\mathfrak{B}(\mathcal{H}, \mathcal{K})$ is the set of all maps $T: \mathcal{H} \rightarrow \mathcal{K}$ for which there is a map $T^{*}: \mathcal{K} \rightarrow \mathcal{H}$ such that $\langle T x, y\rangle=\left\langle x, T^{*} y\right\rangle$ for any $x \in \mathcal{H}$ and $y \in \mathcal{K}$. We know that any element $T$ of $\mathfrak{B}(\mathcal{H}, \mathcal{K})$ is a bounded linear operator. We call $\mathfrak{B}(\mathcal{H}, \mathcal{K})$ the set of adjointable operators from $\mathcal{H}$ into $\mathcal{K}$. In case $\mathcal{H}=\mathcal{K}, \mathfrak{B}(\mathcal{H}, \mathcal{H})$, which we abbreviate to $\mathfrak{B}(\mathcal{H})$, is a $C^{*}$-algebra and we use the notation $I_{\mathcal{H}}$ to denote the identity operator on $\mathcal{H}$. For any $A \in \mathfrak{B}(\mathcal{H}, \mathcal{K})$, the notation $\mathcal{R}(A)$ and $\mathcal{N}(A)$ stand for the range of $A$ and the null space of $A$, respectively. An 
operator $A \in \mathfrak{B}(\mathcal{H}, \mathcal{K})$ is regular if there is an operator $A^{-} \in \mathfrak{B}(\mathcal{K}, \mathcal{H})$ such that $A A^{-} A=A, A^{-}$is called an inner inverse of $A$. It is well known that $A$ is regular if and only if $\mathcal{R}(A)$ and $\mathcal{N}(A)$, respectively, are closed and complemented subspaces of $\mathcal{K}$ and $\mathcal{H}$.

An operator $A \in \mathfrak{B}(\mathcal{H})$ is called Hermitian (or self-adjoint) if $A^{*}=A$, and positive if $\langle A x, x\rangle \geq 0$ for all $x \in H$, we write $A \geq 0$ if $A$ is positive. The set $\mathfrak{B}(\mathcal{H})^{+}$of the positive operators is a subset of the Hermitian operators. For $A \geq 0, A^{\frac{1}{2}}$ denotes the positive operator satisfies $X^{2}=A$. An operator $A \in \mathfrak{U}(\mathcal{H})$ is called unitary if $A^{*} A=A A^{*}=I_{\mathcal{H}}$. The real part (or Hermitian part) of $A \in \mathfrak{B}(\mathcal{H})$ is defined by $\operatorname{Re}(A)=\frac{1}{2}\left(A+A^{*}\right), \operatorname{Re}(A)$ is always Hermitian, $\operatorname{Re}(A)=A$ if $A$ is Hermitian, and $\operatorname{Re}(A)=0$ if $A$ is skew-Hermitian. Further, $\operatorname{Re}(A \pm B)=\operatorname{Re}(A) \pm \operatorname{Re}(B)$, $\operatorname{Re}\left(X^{*} A X\right)=X^{*} \operatorname{Re}(A) X$. An operator $A \in \mathfrak{B}(\mathcal{H})$ is called Re-positive if $\operatorname{Re}(A) \geq$ 0 .

Let $\mathcal{H}, \mathcal{K}$ be two Hilbert $\mathfrak{A}$-modules, $A \in \mathfrak{B}(\mathcal{H}, \mathcal{K})$. The Moore-Penrose inverse $A^{\dagger}$ of $A$ (if it exists) is defined as the unique element of $\mathfrak{B}(\mathcal{K}, \mathcal{H})$ which satisfies the following four Penrose equations

$$
A X A=A, \quad X A X=X,(A X)^{*}=A X,(X A)^{*}=X A .
$$

For any $A \in \mathfrak{B}(\mathcal{H}, \mathcal{K})$, the Moore-Penrose inverse $A^{\dagger}$ of $A$ exists if and only if $A$ has closed range. In this case, $A^{\dagger}$ exists uniquely and $\left(A^{*}\right)^{\dagger}=\left(A^{\dagger}\right)^{*}$. If a regular operator $A$ is positive, then $A^{\dagger} \geq 0$ and $A A^{\dagger}=A^{\dagger} A$. Moreover, both $P_{A}=A^{\dagger} A$ and $Q_{A}=A A^{\dagger}$ are idempotent and self-adjoint. For convenience, we use notations $\mathcal{L}_{A}$ and $\mathcal{R}_{A}$ to stand for $I_{\mathcal{H}}-A^{\dagger} A$ and $I_{\mathcal{K}}-A A^{\dagger}$ induced by $A$, respectively. Obviously, $\mathcal{L}_{A}$ and $\mathcal{R}_{A}$ are also idempotent and self-adjoint and $\mathcal{L}_{A}=\mathcal{R}_{A^{*}}$. For other important properties of operators and generalized inverses of operators, see [10] and [14].

3. Positive solution to system (1.5) of adjointable operator equations. In this Section, we mainly study some necessary and sufficient conditions for system (1.5) of adjointable operator equations to have a positive solution over the Hilbert $C^{*}$-modules. We begin this section with the following lemmas, which can be deduced from $[24]$.

Lemma 3.1. (Theorem 2.1 in [24]) Let $A, C \in \mathfrak{B}\left(\mathcal{H}_{1}, \mathcal{H}_{2}\right), A$ and $C A^{*}$ have closed ranges. Then the adjointable operator equation $A X=C$ has a positive solution $X \in \mathfrak{B}\left(\mathcal{H}_{1}\right)$ if and only if $C A^{*} \geq 0, R(C) \subseteq R\left(C A^{*}\right)$. In this case, the general positive solution is given by

$$
X=C^{*}\left(C A^{*}\right)^{-} C+\mathcal{L}_{A} S \mathcal{L}_{A}^{*}
$$

where $S \in \mathfrak{B}\left(\mathcal{H}_{1}\right)^{+}$is arbitrary, $C^{*}\left(C A^{*}\right)^{-} C$ is a particular positive solution to $A X=$ $C$, independent of the choice of the inner inverse $\left(C A^{*}\right)^{-}$. 
Lemma 3.2. (Theorem 3.7 in [24]) Let $\mathcal{H}, \mathcal{K}, \mathcal{L}$ be Hilbert $C^{*}$-modules, and let $A_{1}, C_{1} \in \mathfrak{B}(\mathcal{H}, \mathcal{K}), B_{2}, C_{2} \in \mathfrak{B}(\mathcal{L}, \mathcal{H})$,

$$
D=\left[\begin{array}{c}
A_{1} \\
B_{2}^{*}
\end{array}\right], \quad E=\left[\begin{array}{c}
C_{1} \\
C_{2}^{*}
\end{array}\right], \quad F=\left[\begin{array}{cc}
C_{1} A_{1}^{*} & C_{1} B_{2} \\
\left(A_{1} C_{2}\right)^{*} & C_{2}^{*} B_{2}
\end{array}\right]
$$

such that $D, F$ are regular. Then system (1.1) has a positive solution $X \in \mathfrak{B}(\mathcal{H})$ if and only if $F$ is positive and $\mathcal{R}(E) \subseteq \mathcal{R}(F)$. In this case, the general positive solution of system (1.1) can be expressed as

$$
X=E^{*} F^{-} E+\mathcal{L}_{D} T \mathcal{L}_{D}^{*},
$$

where $T \in \mathfrak{B}(\mathcal{H})^{+}$is arbitrary.

In 2000, Groß in [9] presented a solvability condition for matrix equation (1.2), and derived an expression for the general positive solution to (1.2), which can be generalized into Hilbert $C^{*}$-module.

Lemma 3.3. (Corollary 2.3 in [9]) Let $H, K$ be Hilbert $C^{*}$-modules. Assume that $A \in \mathfrak{B}(\mathcal{H}, \mathcal{K}), C \in \mathfrak{B}(\mathcal{K})$ such that $A$ has closed range, $C$ is Hermitian and $\mathcal{R}(C) \subseteq \mathcal{R}(A)$. Then equation (1.2) has a positive solution $X \in \mathfrak{B}(\mathcal{H})$ if and only if $C$ is positive. If, in addition, $C$ has closed range, then the general positive solution of (1.2) can be expressed as

$$
X=\left[A^{-} B+\left(I_{n}-A^{-} A\right) Y\right]\left[A^{-} B+\left(I_{n}-A^{-} A\right) Y\right]^{*}
$$

where $Y$ is an arbitrary operator in $\mathfrak{B}(\mathcal{H}), B$ is an arbitrary operator with $C=B B^{*}$.

It is easy to verify the following Lemma.

Lemma 3.4. Given operators $A, C \in \mathfrak{B}(\mathcal{H}, \mathcal{K})$. Then $A^{*} A=C^{*} C$ if and only if $A=C T$ for some unitary operator $T$.

Theorem 3.5. Let $A_{1}, C_{1} \in \mathfrak{B}\left(\mathcal{H}, \mathcal{K}_{1}\right), B_{2}, C_{2} \in \mathfrak{B}\left(\mathcal{K}_{2}, \mathcal{H}\right), A_{3} \in \mathfrak{B}\left(\mathcal{H}, \mathcal{K}_{3}\right)$, $A_{4} \in \mathfrak{B}\left(\mathcal{H}, \mathcal{K}_{4}\right), C_{3} \in \mathfrak{B}\left(\mathcal{K}_{3}\right), C_{4} \in \mathfrak{B}\left(\mathcal{K}_{4}\right)$ be given such that system of adjointable operator equations

$$
A_{1} X=C_{1}, \quad X B_{2}=C_{2}, \quad A_{3} X A_{3}^{*}=C_{3}, \quad A_{4} X A_{4}^{*}=C_{4}
$$

is consistent. Denote

$$
\begin{gathered}
M=\left[\begin{array}{cc}
C_{1} A_{1}^{*} & C_{1} B_{2} \\
C_{2}^{*} A_{1}^{*} & C_{2}^{*} B_{2}
\end{array}\right], X_{0}=\left[\begin{array}{ll}
C_{1}^{*} & C_{2}
\end{array}\right] M^{-}\left[\begin{array}{c}
C_{1} \\
C_{2}^{*}
\end{array}\right], \quad A_{11}=\left[\begin{array}{c}
A_{1} \\
B_{2}^{*}
\end{array}\right], \\
C_{33}=C_{3}-A_{3} X_{0} A_{3}^{*}, \quad C_{44}=C_{4}-A_{4} X_{0} A_{4}^{*}, \quad A_{33}=A_{3} L_{A_{11}}, \quad A_{44}=A_{4} L_{A_{11}} .
\end{gathered}
$$


Suppose that $A_{1}, B_{2}, A_{3}, A_{4}, A_{33}, A_{44}, C_{33}, C_{44}$ and $M$ have closed ranges. Then the following statements are equivalent:

(1) the consistent system (3.1) of adjointable operator equations has a positive solution in $\mathfrak{B}(\mathcal{H})^{+}$,

(2) $M, C_{3}, C_{4}, C_{33}, C_{44}$ are positive,

$$
\mathcal{R}\left(\left[\begin{array}{l}
C_{1} \\
C_{2}^{*}
\end{array}\right]\right) \subseteq \mathcal{R}(M), \quad \mathcal{R}\left(C_{33}^{\frac{1}{2}}\right) \subseteq \mathcal{R}\left(A_{33}\right), \quad \mathcal{R}\left(C_{44}^{\frac{1}{2}}\right) \subseteq \mathcal{R}\left(A_{44}\right),
$$

and there exist an unitary operator $T$ such that

$$
A_{44} \mathcal{L}_{A_{33}}\left(A_{44} \mathcal{L}_{A_{33}}\right)^{-}\left(C_{44}^{\frac{1}{2}} T-A_{44} A_{33}^{-} C_{33}^{\frac{1}{2}}\right)=\left(C_{44}^{\frac{1}{2}} T-A_{44} A_{33}^{-} C_{33}^{\frac{1}{2}}\right) .
$$

In this case, the general positive solutions can be expressed as

$$
X=X_{0}+\mathcal{L}_{A_{11}}\left(A_{33}^{-} C_{33}^{\frac{1}{2}}+\mathcal{L}_{A_{33}} Y\right)\left(A_{33}^{-} C_{33}^{\frac{1}{2}}+\mathcal{L}_{A_{33}} Y\right)^{*}\left(\mathcal{L}_{A_{11}}\right)^{*}
$$

where

$$
Y=\left(A_{44} \mathcal{L}_{A_{33}}\right)^{-}\left(C_{44}^{\frac{1}{2}} T-A_{44} A_{33}^{-} C_{33}^{\frac{1}{2}}\right)+W-\left(A_{44} \mathcal{L}_{A_{33}}\right)^{-}\left(A_{44} \mathcal{L}_{A_{33}}\right) W,
$$

with $W$ is free to vary over $\mathfrak{B}\left(\mathcal{H}, \mathcal{K}_{1}\right)$.

Proof. $(1) \Rightarrow(2)$. Suppose that system (3.1) of adjointable operator equations has a positive solution $X$, then it follows from Lemma 3.2-3.3 that $C_{3}, C_{4}, M$ are positive, and

$$
\mathcal{R}\left(\left[\begin{array}{c}
C_{1} \\
C_{2}^{*}
\end{array}\right]\right) \subseteq \mathcal{R}(M)
$$

Obviously, $X$ satisfies system of adjointable operator equations

$$
A_{1} X=C_{1}, \quad X B_{2}=C_{2},
$$

thus there exist an operator $V \in \mathfrak{B}\left(H_{1}\right)^{+}$such that

$$
X=X_{0}+\mathcal{L}_{D} V \mathcal{L}_{D}^{*}
$$

Taking (3.7) into $A_{3} X A_{3}^{*}=C_{3}$ yields that

$$
A_{3} \mathcal{L}_{A_{11}} V\left(A_{3} \mathcal{L}_{A_{11}}\right)^{*}=C_{3}-A_{3} X_{0} A_{3}^{*}
$$

has a positive solution with respect to $V$. By Lemma 3.3, we have

$$
C_{33}=C_{3}-A_{3} X_{0} A_{3}^{*} \geq 0, \quad \mathcal{R}\left(C_{33}^{\frac{1}{2}}\right) \subseteq \mathcal{R}\left(A_{3} \mathcal{L}_{A_{11}}\right)=\mathcal{R}\left(A_{33}\right),
$$


and $V$ can be expressed as

$$
V=\left(A_{33}^{-} C_{33}^{\frac{1}{2}}+\mathcal{L}_{A_{33}} Y\right)\left(A_{33}^{-} C_{33}^{\frac{1}{2}}+\mathcal{L}_{A_{33}} Y\right)^{*}
$$

with some $Y \in \mathfrak{B}\left(\mathcal{H}, \mathcal{K}_{1}\right)$. Taking (3.7) with (3.9) into $A_{4} X A_{4}^{*}=C_{4}$ gives

$$
A_{4}\left(X_{0}+\mathcal{L}_{A_{11}}\left(A_{33}^{-} C_{33}^{\frac{1}{2}}+\mathcal{L}_{A_{33}} Y\right)\left(A_{33}^{-} C_{33}^{\frac{1}{2}}+\mathcal{L}_{A_{33}} Y\right)^{*}\left(\mathcal{L}_{A_{11}}\right)^{*}\right) A_{4}^{*}=C_{4}
$$

Then $C_{44}=C_{4}-A_{4} X_{0} A_{4}^{*} \geq 0$ and $\mathcal{R}\left(C_{44}^{\frac{1}{2}}\right) \subseteq \mathcal{R}\left(A_{4} \mathcal{L}_{A_{11}}\right)=\mathcal{R}\left(A_{44}\right)$. Moreover, it follows Lemma 3.4 that there exist an unitary operator $T$ such that

$$
A_{44}\left(A_{33}^{-} C_{33}^{\frac{1}{2}}+\mathcal{L}_{A_{33}} Y\right)=C_{44}^{\frac{1}{2}} T \text {. }
$$

Then equation

$$
A_{44} \mathcal{L}_{A_{33}} Y=C_{44}^{\frac{1}{2}} T-A_{44} A_{33}^{-} C_{33}^{\frac{1}{2}}
$$

is consistent with respect to $Y$, from which we can get $(3.3)$.

$(2) \Rightarrow(1)$. Since $M$ is positive and $\mathcal{R}(M) \subseteq \mathcal{R}\left(\left[\begin{array}{l}C_{1} \\ C_{2}^{*}\end{array}\right]\right)$, then system (3.6) has a positive solution which can be expressed as (3.7). Taking (3.7) into $A_{3} X A_{3}^{*}=C_{3}$, and combining $C_{33}, C_{44}$ are positive, $\mathcal{R}\left(C_{33}^{\frac{1}{2}}\right) \subseteq \mathcal{R}\left(A_{33}\right)$, we have equation (3.8) has a positive solution with respect to $V$, which can be written as

$$
V=\left(A_{33}^{-} C_{33}^{\frac{1}{2}}+\mathcal{L}_{A_{33}} Y\right)\left(A_{33}^{-} C_{33}^{\frac{1}{2}}+\mathcal{L}_{A_{33}} Y\right)^{*}
$$

Thus, equations

$$
A_{1} X=C_{1}, \quad X B_{2}=C_{2}, \quad A_{3} X A_{3}^{*}=C_{3}
$$

have a common positive solution

$$
\begin{aligned}
X & =\left[\begin{array}{ll}
C_{1}^{*} & C_{2}
\end{array}\right] M^{-}\left[\begin{array}{c}
C_{1} \\
C_{2}^{*}
\end{array}\right] \\
& +\mathcal{L}_{A_{11}}\left(A_{33}^{-} C_{33}^{\frac{1}{2}}+\mathcal{L}_{A_{33}} Y\right)\left(A_{33}^{-} C_{33}^{\frac{1}{2}}+\mathcal{L}_{A_{33}} Y\right)^{*} \mathcal{L}_{A_{11}}^{*},
\end{aligned}
$$

where $Y$ free to vary over $\mathfrak{B}\left(\mathcal{H}, \mathcal{K}_{1}\right)$. Next we will show that (3.10) with (3.5) satisfies $A_{4} X A_{4}^{*}=C_{4}$. Choosing $Y$ as (3.5) and by direct computation we have

$$
\begin{aligned}
& A_{4}\left(X_{0}+\mathcal{L}_{A_{11}}\left(A_{33}^{-} C_{33}^{\frac{1}{2}}+\mathcal{L}_{A_{33}} Y\right)\left(A_{33}^{-} C_{33}^{\frac{1}{2}}+\mathcal{L}_{A_{33}} Y\right)^{*}\left(\mathcal{L}_{A_{11}}\right)^{*}\right) A_{4}^{*} \\
= & A_{4} X_{0} A_{4}^{*}+\left(A_{44}\left(A_{33}^{-} C_{33}^{\frac{1}{2}}+\mathcal{L}_{A_{33}} Y\right)\right)\left(A_{44}\left(A_{33}^{-} C_{33}^{\frac{1}{2}}+\mathcal{L}_{A_{33}} Y\right)\right)^{*} \\
= & A_{4} X_{0} A_{4}^{*}+C_{44}^{\frac{1}{2}} T\left(C_{44}^{\frac{1}{2}} T\right)^{*}=C_{4} .
\end{aligned}
$$


Therefore, (3.10) is a positive solution to system (3.1).

From the above proofs, we get that (3.4) with (3.5) is a positive solution to system (3.1). Next we show that every positive solution of system (3.1) can be expressed as (3.4) with (3.5). Suppose that $X_{1}$ is an arbitrary positive solution of system (3.1), then $X_{1}$ is a positive solution of system of adjointable operator equations (3.6). It follows from Lemma 3.2 that there exists a positive $V_{0}$ such that

$$
X_{1}=\left[\begin{array}{ll}
C_{1}^{*} & C_{2}
\end{array}\right] M^{-}\left[\begin{array}{c}
C_{1} \\
C_{2}^{*}
\end{array}\right]+\mathcal{L}_{A_{11}} V_{0} \mathcal{L}_{A_{11}}^{*} .
$$

Setting $W=V_{0}^{\frac{1}{2}}$, we have

$$
Y=\left(A_{44} \mathcal{L}_{A_{33}}\right)^{-}\left(C_{44}^{\frac{1}{2}} T-A_{44} A_{33}^{-} C_{33}^{\frac{1}{2}}\right)+V_{0}^{\frac{1}{2}}-\left(A_{44} \mathcal{L}_{A_{33}}\right)^{-}\left(A_{44} \mathcal{L}_{A_{33}}\right) V_{0}^{\frac{1}{2}}=V_{0}^{\frac{1}{2}} .
$$

Taking it into (3.4), we can get

$X_{1}=\left[\begin{array}{ll}C_{1}^{*} & C_{2}\end{array}\right] M^{-}\left[\begin{array}{c}C_{1} \\ C_{2}^{*}\end{array}\right]+\mathcal{L}_{A_{11}}\left(A_{33}^{-} C_{33}^{\frac{1}{2}}+\mathcal{L}_{A_{33}} V_{0}^{\frac{1}{2}}\right)\left(A_{33}^{-} C_{33}^{\frac{1}{2}}+\mathcal{L}_{A_{33}} V_{0}^{\frac{1}{2}}\right)^{*}\left(\mathcal{L}_{A_{11}}\right)^{*}$.

Thus, (3.4) with (3.5) is the general positive solution of system (3.1).

We now can consider a special case of Theorem 3.5.

Corollary 3.6. Let $A_{3} \in \mathfrak{B}\left(\mathcal{H}, \mathcal{K}_{3}\right), A_{4} \in \mathfrak{B}\left(\mathcal{H}, \mathcal{K}_{4}\right), C_{3} \in \mathfrak{B}\left(\mathcal{K}_{3}\right)$ and $C_{4} \in$ $\mathfrak{B}\left(\mathcal{K}_{4}\right)$ be given such that system of adjointable operator equations

$$
A_{3} X A_{3}^{*}=C_{3}, \quad A_{4} X A_{4}^{*}=C_{4}
$$

is consistent. Suppose $A_{3}$ and $A_{4}$ have closed range. Then the following statements are equivalent:

(1) System (3.11) has a positive solution.

(2) $C_{3}, C_{4}$ are positive

$$
\mathcal{R}\left(C_{3}^{\frac{1}{2}}\right) \subseteq \mathcal{R}\left(A_{3}\right), \quad \mathcal{R}\left(C_{3}^{\frac{1}{2}}\right) \subseteq \mathcal{R}\left(A_{4}\right),
$$

and there exist a $T \in \mathfrak{U}(\mathcal{H})$ such that

$$
\left(A_{4} L_{A_{3}}\right)^{-} A_{4} L_{A_{3}}\left(C_{4}^{\frac{1}{2}} T-A_{4} A_{3}^{-} C_{3}^{\frac{1}{2}}\right)=\left(C_{4}^{\frac{1}{2}} T-A_{4} A_{3}^{-} C_{3}^{\frac{1}{2}}\right) .
$$

In this case, the general positive solution of system (3.11) can be expressed as

$$
X=\left(A_{3}^{-} C_{3}^{\frac{1}{2}}+\mathcal{L}_{A_{3}} Y\right)\left(A_{3}^{-} C_{3}^{\frac{1}{2}}+\mathcal{L}_{A_{3}} Y\right)^{*}
$$

with

$$
Y=\left(A_{4} \mathcal{L}_{A_{3}}\right)^{-}\left(C_{4}^{\frac{1}{2}} T-A_{4} A_{3}^{-} C_{3}^{\frac{1}{2}}\right)+W-\left(A_{4} \mathcal{L}_{A_{3}}\right)^{-} A_{4} \mathcal{L}_{A_{3}} W
$$


where $W$ is free to vary over $\mathfrak{B}(\mathcal{H})$.

Remark 3.7. For finite complex matrices, Theorem 3.5 can be seen as a complementarity of Zhang [26] by adding the proof that every positive solution of system (3.11) can be expressed as the form (3.13) with proper choice of $W$.

4. Re-positive solution to system (1.1) of adjointable operator equations. In order to establish some necessary and sufficient conditions for system (1.1) of adjointable operator equations to have a Re-positive solution over the Hilbert $C^{*}$ modules, we need the following lemma which is due to Wang et al. [17].

Lemma 4.1. Let $A \in \mathfrak{B}\left(\mathcal{K}_{1}, \mathcal{H}\right), B \in \mathfrak{B}\left(\mathcal{H}, \mathcal{K}_{2}\right), C \in \mathfrak{B}\left(\mathcal{K}_{3}, \mathcal{H}\right)$ and $D \in \mathfrak{B}\left(\mathcal{H}, \mathcal{K}_{4}\right)$ be given. Denote $M=R_{A} C, N=D L_{B}$ and $S=C L_{M}$. Suppose that $A, B, C, D, E$, $M, N, S, R_{A} E$ and $E L_{B}$ have closed ranges. Then

(a) there exist $X \in \mathfrak{B}\left(\mathcal{K}_{2}, \mathcal{K}_{1}\right), Y \in \mathfrak{B}\left(\mathcal{K}_{3}, \mathcal{K}_{4}\right)$ such that

$$
A X B+C Y D=E
$$

if and only if

$$
\begin{aligned}
& \mathcal{R}(E) \subseteq \mathcal{R}\left[\begin{array}{ll}
A & C
\end{array}\right], \quad \mathcal{R}\left(E^{*}\right) \subseteq \mathcal{R}\left(\left[\begin{array}{ll}
B^{*} & D^{*}
\end{array}\right]\right)^{*}, \\
& \mathcal{R}(E) \subseteq \mathcal{R}(A) \oplus \mathcal{R}\left(D^{*}\right), \quad \mathcal{R}(E) \subseteq \mathcal{R}(C) \oplus \mathcal{R}\left(B^{*}\right),
\end{aligned}
$$

or equivalently,

$$
R_{M} R_{A} E=0, R_{A} E L_{D}=0, E L_{B} L_{N}=0, R_{C} E L_{B}=0 .
$$

In this case, the general solution can be expressed as

$X=A^{\dagger} E B^{\dagger}-A^{\dagger} C M^{\dagger} R_{A} E B^{\dagger}-A^{\dagger} S C^{\dagger} E L_{B} N^{\dagger} D B^{\dagger}-A^{\dagger} S V R_{N} D B^{\dagger}+L_{A} U+Z R_{B}$, $Y=M^{\dagger} R_{A} E D^{\dagger}+L_{M} S^{\dagger} S C^{\dagger} E L_{B} N^{\dagger}+L_{M}\left(V-S^{\dagger} S V N N^{\dagger}\right)+W R_{D}$,

where $U, V, W, Z$ are arbitrary.

Lemma 4.2. Let $A \in \mathfrak{B}\left(\mathcal{K}_{1}, \mathcal{H}\right), B \in \mathfrak{B}\left(\mathcal{H}, \mathcal{K}_{2}\right)$ and $C \in \mathfrak{B}(\mathcal{H})$ be given. Denote $M=R_{A} B^{*}, N=A^{*} L_{B}$ and $S=B^{*} L_{M}$. Suppose that $A, B, C, M, N$ and $S$ have closed ranges, then

(a) there exist an $X \in \mathfrak{B}\left(\mathcal{K}_{2}, \mathcal{K}_{1}\right)$ such that

$$
A X B+(A X B)^{*}=C,
$$

if and only if

$$
C^{*}=C, \mathcal{R}(C) \subseteq \mathcal{R}\left[\begin{array}{ll}
A & B^{*}
\end{array}\right],
$$




$$
\mathcal{R}(C) \subseteq \mathcal{R}(A) \oplus \mathcal{R}\left(A^{*}\right), \quad \mathcal{R}(C) \subseteq \mathcal{R}(B) \oplus \mathcal{R}\left(B^{*}\right)
$$

or equivalently,

$$
C^{*}=C, \quad\left[\begin{array}{ll}
A & B^{*}
\end{array}\right]\left[\begin{array}{ll}
A & B^{*}
\end{array}\right]^{\dagger} C=C
$$

and $R_{A} C R_{A}=0, L_{B} C L_{B}=0$.

(b) In this case, the general solution of (4.1) can be expressed as

$$
X=\frac{1}{2}\left(U+V^{*}\right),
$$

where $U$ and $V$ are the general solutions of equation

$$
A U B+B^{*} V A^{*}=C .
$$

Written in an explicit form

$$
\begin{aligned}
U & =A^{\dagger} C B^{\dagger}-\left(B^{*}\right)^{\dagger} M^{\dagger} R_{A} C B^{\dagger} \\
& -A^{\dagger} S\left(B^{*}\right)^{\dagger} C L_{B} N^{\dagger} A^{*} B^{\dagger}-A^{\dagger} S V R_{N} A^{*} B^{\dagger}+L_{A} U+Z R_{B}, \\
V & =M^{\dagger} R_{A} C\left(A^{*}\right)^{\dagger}+L_{M} S^{\dagger} S\left(B^{*}\right)^{\dagger} C L_{B} N^{\dagger} \\
& +L_{M}\left(V-S^{\dagger} S V N N^{\dagger}\right)+W R_{A^{*}},
\end{aligned}
$$

where $U, V, W, Z$ are arbitrary.

Proof. Suppose $X$ satisfies (4.1). Then it is easy to see that $C$ is Hermitian. On the other hand, if there is an $X$ satisfying (4.1), then there are $X, Y$ satisfying $A X B+B^{*} Y A^{*}=C$. Hence by Lemma 4.1, we have

$$
\mathcal{R}(C) \subseteq \mathcal{R}\left[\begin{array}{ll}
A & B^{*}
\end{array}\right], \mathcal{R}(C) \subseteq \mathcal{R}(A) \oplus \mathcal{R}\left(A^{*}\right), \quad \mathcal{R}(C) \subseteq \mathcal{R}(B) \oplus \mathcal{R}\left(B^{*}\right) .
$$

Conversely, if (4.2) and (4.3) hold, then there exist $U$ and $V$ such that

$$
A U B+B^{*} V A^{*}=C .
$$

Taking conjugate transpose for this equality gives

$$
B^{*} U^{*} A^{*}+A V^{*} B=C .
$$

Adding these two equalities and dividing by 2 gives

$$
A\left(\frac{U+V^{*}}{2}\right) B+B^{*}\left(\frac{U+V^{*}}{2}\right)^{*} A^{*}=C .
$$

This equality implies that for any pair of solutions of $A U B+B^{*} V A^{*}=C$ the expression (4.4) is a solution to (4.1). Suppose $X_{0}$ is any solution to (4.1). Then $A U B+B^{*} V A^{*}=C$ has solutions $U=V^{*}=X_{0}$. Thus, $X_{0}$ can be expressed as

$$
X_{0}=\frac{1}{2}\left(X_{0}+X_{0}\right)=\frac{1}{2}\left(U+V^{*}\right) .
$$


From Lemma 4.1, the general solutions of can be written as (4.4) with (4.5) and (4.6).

Theorem 4.3. Let $A, C \in \mathfrak{B}\left(\mathcal{H}, \mathcal{K}_{1}\right), B, D \in \mathfrak{B}\left(\mathcal{K}_{2}, \mathcal{H}\right)$ be given such that system (1.1) is consistent. For simplicity, put

$$
\begin{aligned}
& X_{0}=A^{\dagger} C+D B^{\dagger}-A^{\dagger} A D B^{\dagger}, \quad J=\left[\begin{array}{ll}
L_{A} & R_{B}
\end{array}\right], M=P_{A} R_{B}, \\
& N=L_{A} Q_{B}, \quad S=R_{B} L_{M} A_{33}=P_{A} P_{J}, A_{44}=Q_{B} P_{J}, \\
& H_{0}=\left(X_{0}+X_{0}^{*}\right) R_{J}\left(R_{J}\left(X_{0}+X_{0}^{*}\right) R_{J}\right)^{\dagger} R_{J}\left(X_{0}+X_{0}^{*}\right), \\
& C_{3}=P_{A}\left(X_{0}+X_{0}^{*}\right) P_{A}, C_{4}=Q_{B}\left(X_{0}+X_{0}^{*}\right) Q_{B}, \quad G=R_{J}\left(X_{0}+X_{0}^{*}\right) R_{J}, \\
& Z=R_{J}\left(X_{0}+X_{0}^{*}\right) R_{J}, C_{33}=C_{3}-P_{A} R_{J} Z^{\dagger} R_{J} P_{A}, C_{44}=C_{4}-Q_{B} R_{J} Z^{\dagger} R_{J} Q_{B}, \\
& C_{5}=C_{3}-P_{A} H_{0} P_{A}, C_{6}=C_{4}-Q_{B} H_{0} Q_{B} .
\end{aligned}
$$

Suppose $J, M, N, S, A_{33}$ and $A_{44}$ have closed ranges. Then the following statements are equivalent:

(1) system (1.1) has a Re-positive solution,

(2) $C_{3}, C_{4}, G, C_{33}$ and $C_{44}$ are positive,

$$
\mathcal{R}\left(R_{J}\left(X_{0}+X_{0}^{*}\right) R_{J}\right) \subseteq \mathcal{R}\left(R_{J}\left(X_{0}+X_{0}^{*}\right)\right), \mathcal{R}\left(C_{33}^{\frac{1}{2}}\right) \subseteq \mathcal{R}\left(A_{33}\right), \mathcal{R}\left(C_{44}^{\frac{1}{2}}\right) \subseteq \mathcal{R}\left(A_{44}\right)
$$

and there exist a $T \in \mathfrak{U}(\mathcal{H})$ such that

$$
A_{44} L_{A_{33}}\left(A_{44} L_{A_{33}}\right)^{\dagger}\left(C_{44}^{\frac{1}{2}} T-A_{44} A_{33}^{\dagger} C_{33}^{\frac{1}{2}}\right)=\left(C_{44}^{\frac{1}{2}} T-A_{44}\left(A_{33}\right)^{\dagger} C_{33}^{\frac{1}{2}}\right) .
$$

In this case, the general Re-positive solution to system (1.1) can be expressed as

$$
X=X_{0}+\frac{1}{2} L_{A}\left(U+Z^{*}\right) R_{B}
$$

where

$$
\begin{aligned}
U & =L_{A}\left(H-\left(X_{0}+X_{0}^{*}\right)\right) R_{B}-R_{B} M^{\dagger} P_{A}\left(H-\left(X_{0}+X_{0}^{*}\right)\right) R_{B} \\
& -L_{A} S R_{B}\left(H-\left(X_{0}+X_{0}^{*}\right)\right) Q_{B} N^{\dagger} L_{A} R_{B} \\
& -L_{A} S W_{1} R_{N} L_{A} R_{B}+P_{A} W_{2}+W_{3} Q_{B}, \\
Z & =M^{\dagger} P_{A}\left(H-\left(X_{0}+X_{0}^{*}\right)\right) L_{A}+L_{M} S^{\dagger} S R_{B}\left(H-\left(X_{0}+X_{0}^{*}\right)\right) Q_{B} N^{\dagger} \\
& +L_{M}\left(W_{1}-S^{\dagger} S W_{1} N N^{\dagger}\right)+W_{4} P_{A},
\end{aligned}
$$

with

$$
H=H_{0}+Q_{J}\left(\left(P_{A} Q_{J}\right)^{\dagger} C_{5}^{\frac{1}{2}}+L_{P_{A} P_{J}} Y\right)\left(\left(P_{A} Q_{J}\right)^{\dagger} C_{5}^{\frac{1}{2}}+L_{P_{A} P_{J}} Y\right)^{*} Q_{J}
$$

and

$$
Y=\left(Q_{B} Q_{J} P_{A} Q_{J}\right)^{-}\left(C_{6}^{\frac{1}{2}} T-Q_{B} Q_{J}\left(P_{A} Q_{J}\right)^{-} C_{5}^{\frac{1}{2}}\right)+L_{Q_{B} Q_{J} P_{A} Q_{J}} W_{5},
$$


$W_{i}, i=1, \ldots, 5$, be arbitrary.

Proof. It is easy to verify that the general solution of (1.1) can be expressed as

$$
X=X_{0}+L_{A} V R_{B}
$$

where $X_{0}=A^{\dagger} C+D B^{\dagger}-A^{\dagger} A D B^{\dagger}, V$ is free to vary over $\mathfrak{B}\left(\mathcal{K}_{2}, \mathcal{H}\right)$. Note that

$$
X+X^{*}=X_{0}+X_{0}^{*}+L_{A} V R_{B}+\left(L_{A} V R_{B}\right)^{*}=H .
$$

Hence,

$$
X+X^{*} \geq 0 \Leftrightarrow H \geq 0
$$

Fix $H$ first and it is easy to get $H=H^{*}$. From Lemma 4.1, equation

$$
L_{A} V R_{B}+\left(L_{A} V R_{B}\right)^{*}=H-\left(X_{0}+X_{0}^{*}\right)
$$

is consistent for $V$ if and only if

$$
\mathcal{R}\left(H-X_{0}-X_{0}^{*}\right) \subseteq \mathcal{R}\left[\begin{array}{ll}
L_{A} & R_{B}
\end{array}\right], \quad \mathcal{R}\left(H-X_{0}-X_{0}^{*}\right) \subseteq \mathcal{R}\left(L_{A}\right) \oplus \mathcal{R}\left(\left(L_{A}\right)^{*}\right)
$$

and

$$
\mathcal{R}\left(H-X_{0}-X_{0}^{*}\right) \subseteq \mathcal{R}\left(R_{B}\right) \oplus \mathcal{R}\left(\left(R_{B}\right)^{*}\right),
$$

which are equivalent to the following system

$$
\left\{\begin{array} { c } 
{ R _ { J } ( H - X _ { 0 } - X _ { 0 } ^ { * } ) = 0 } \\
{ P _ { A } ( H - X _ { 0 } - X _ { 0 } ^ { * } ) P _ { A } = 0 } \\
{ Q _ { B } ( H - X _ { 0 } - X _ { 0 } ^ { * } ) Q _ { B } = 0 }
\end{array} \Leftrightarrow \left\{\begin{array}{c}
R_{J} H=R_{J}\left(X_{0}+X_{0}^{*}\right) \\
P_{A} H P_{A}=P_{A}\left(X_{0}+X_{0}^{*}\right) P_{A} \\
Q_{B} H Q_{B}=Q_{B}\left(X_{0}+X_{0}^{*}\right) Q_{B}
\end{array}\right.\right.
$$

has a positive solution $H$. Next we will show the equivalence between (1) and (2).

$(1) \Rightarrow(2)$. Suppose that system (1.1) has a Re-positive solution $X_{1}$. Then it follows from the above proof that system (4.13) has a positive solution. Noticing Theorem 3.5, we have

$$
\begin{aligned}
& R_{J}\left(X_{0}+X_{0}^{*}\right) R_{J}, \quad P_{A}\left(X_{0}+X_{0}^{*}\right) P_{A}, \quad Q_{B}\left(X_{0}+X_{0}^{*}\right) Q_{B}, \\
& C_{3}-P_{A} R_{J} Z^{\dagger} R_{J} P_{A}, \quad C_{4}-Q_{B} R_{J} Z^{\dagger} R_{J} Q_{B}
\end{aligned}
$$

are positive,

$$
\mathcal{R}\left(R_{J}\left(X_{0}+X_{0}^{*}\right) R_{J}\right) \subset \mathcal{R}\left(R_{J}\left(X_{0}+X_{0}^{*}\right)\right), \mathcal{R}\left(C_{33}^{\frac{1}{2}}\right) \subseteq \mathcal{R}\left(A_{33}\right), \mathcal{R}\left(C_{44}^{\frac{1}{2}}\right) \subseteq \mathcal{R}\left(A_{44}\right),
$$

and there exist a $T \in \mathfrak{U}(\mathcal{H})$ such that

$$
A_{44} L_{A_{33}}\left(A_{44} L_{A_{33}}\right)^{\dagger}\left(C_{44}^{\frac{1}{2}} T-A_{44} A_{33}^{\dagger} C_{33}^{\frac{1}{2}}\right)=\left(C_{44}^{\frac{1}{2}} T-A_{44}\left(A_{33}\right)^{\dagger} C_{33}^{\frac{1}{2}}\right) .
$$


$(2) \Rightarrow(1)$. Note that the operators in (4.14) are all positive and equations (4.15)(4.16) are satisfied. By Theorem 3.5, system (4.13) has a positive solution $H$ which can be expressed as

$$
H=H_{0}+Q_{J} K\left(Q_{J} K\right)^{*}
$$

where

$$
K=\left(P_{A} Q_{J}\right)^{-}\left(P_{A}\left(X_{0}+X_{0}^{*}\right) P_{A}-P_{A} H_{0} P_{A}\right)^{\frac{1}{2}}+L_{P_{A} P_{J}} Y
$$

with

$$
\begin{aligned}
Y= & \left(Q_{B} Q_{J} P_{A} Q_{J}\right)^{-}\left(\left(C_{4}-Q_{B} H_{0} Q_{B}\right)^{\frac{1}{2}} T-Q_{B} Q_{J}\left(P_{A} Q_{J}\right)^{-}\left(C_{3}-P_{A} H_{0} P_{A}\right)^{\frac{1}{2}}\right) \\
& +L_{Q_{B} Q_{J} P_{A} Q_{J}} W_{5},
\end{aligned}
$$

where $W_{5}$ is free to vary over $\mathfrak{B}\left(\mathcal{H}, \mathcal{K}_{1}\right)$. For arbitrary $H$ satisfies (4.13) and noting Lemma 4.1, we can get the general solution to equation (4.12) relate to $V$ can be written as $V=\frac{1}{2}\left(U+Z^{*}\right)$ with

$$
\begin{aligned}
U & =L_{A}\left(H-\left(X_{0}+X_{0}^{*}\right)\right) R_{B}-R_{B} M^{\dagger} P_{A}\left(H-\left(X_{0}+X_{0}^{*}\right)\right) R_{B} \\
& -L_{A} S R_{B}\left(H-\left(X_{0}+X_{0}^{*}\right)\right) Q_{B} N^{\dagger} L_{A} R_{B}-L_{A} S W_{1} R_{N} L_{A} R_{B}+P_{A} W_{2}+W_{3} Q_{B}, \\
Z & =M^{\dagger} P_{A}\left(H-\left(X_{0}+X_{0}^{*}\right)\right) L_{A}+L_{M} S^{\dagger} S R_{B}\left(H-\left(X_{0}+X_{0}^{*}\right)\right) Q_{B} N^{\dagger} \\
& +L_{M}\left(W_{1}-S^{\dagger} S W_{1} N N^{\dagger}\right)+W_{4} P_{A},
\end{aligned}
$$

where $W_{i}, i=1, \ldots, 4$ are arbitrary. Then the general Re-positive solution of system (1.1) can be expressed as (4.7) with (4.8)-(4.11).

Corollary 4.4. Let $A, C \in \mathfrak{B}\left(\mathcal{H}, \mathcal{K}_{1}\right)$ be given such that equation (1.4) is consistent. Then (1.4) has a real positive solution if and only if $P_{A}\left(A^{\dagger} C+\left(A^{\dagger} C\right)^{*}\right) P_{A}$ is positive. In this case, the general real positive solution to equation (1.4) can be expressed as

$$
X=A^{\dagger} C+\frac{1}{2} L_{A}\left(H-A^{\dagger} C-\left(A^{\dagger} C\right)^{*}\right)\left(I+A^{\dagger} A\right)
$$

with

$$
H=P_{A}^{=}\left(P_{A}\left(A^{\dagger} C+\left(A^{\dagger} C\right)^{*}\right) P_{A}\right) P_{A}^{=}+L_{A} U L_{A},
$$

where

$$
P_{A}^{=}=P_{A}+L_{A} Z\left(P_{A}\left(A^{\dagger} C+\left(A^{\dagger} C\right)^{*}\right) P_{A}\right)^{\frac{1}{2}},
$$

$Z$ is free to vary over $\mathfrak{B}\left(\mathcal{K}_{1}, \mathcal{H}\right)$ and $U \in \mathfrak{B}(\mathcal{H})^{+}$is arbitrary. 
REMARK 4.5. Groß [8] presented a necessary and sufficient condition for the existence of Re-positive solution to matrix equation (1.4) and established the expression of the general Re-positive solution in terms of generalized inverse of some matrices when the solvability conditions are satisfied. However, in 2008, Dajić and Koliha [5] pointed out that the general expression of Re-positive solution to matrix equation (1.4) in [8] did not involve all of the Re-positive solution. They also gave a correct expression of the general Re-positive solution to operator equation (1.4) in terms of generalized inverses of some adjointable operators [5, Theorem 7.3]. In Corollary 4.4, we also give a new expression of this general Re-positive solution which is different from one in $[5]$.

5. Conclusion. In this paper, we have established some necessary and sufficient conditions for system (1.5) and system (1.1) to have a positive or Re-positive solution, respectively, and derived some expressions of the general positive or Re-positive solutions to these systems when the conditions are satisfied.

It is worthy to say that the approach and results in this paper are also true to the bounded operators between quaternionic Hilbert spaces, which plays an important role in certain physical problems (see, for example, [7]).

Motivated by the work in this paper, it would be of interest to investigate the common Re-positive solutions to equations (1.3) for Hilbert $C^{*}$-modules operators.

\section{REFERENCES}

[1] J.K. Baksalary. Nonnegative definite and positive definite solutions to the matrix equation $A X A^{*}=B$. Linear Multilinear Algebra, 16:133-139, 1984.

[2] D.S. Cvetković-Ilić, A. Dajić, and J.J. Koliha. Positive and real-positive solutions to the equation $a x a^{*}=c$ in $C^{*}$-algebras. Linear Multilinear Algebra, 55:535-543, 2007.

[3] D.S. Cvetković-Ilić. Re-nnd solution of the matrix equation $A X B=C$. J. Aust. Math. Soc., 84:63-72, 2008.

[4] A. Dajić and J.J. Koliha. Positive solutions to the equations $A X=C$ and $X B=D$ for Hilbert space operators. J. Math. Anal. Appl., 333:567-576, 2007.

[5] A. Dajić and J.J. Koliha. Equations $a x=c$ and $x b=d$ in rings and rings with involution with applications to Hilbert space operators. Linear Algebra Appl., 429:1779-1809, 2008.

[6] X. Fang, J. Yu, and H. Yao. Solutions to operator equations on Hilbert $C^{*}$-modules. Linear Algebra Appl., 431:2142-2153, 2009.

[7] D. Finkelstein, J.M. Jauch, S. Schiminovich, and D. Speiser. Foundations of quaternion quantum mechanics. J. Math. Phys., 3:207-220, 1962.

[8] J. Groß. Explicit solutions to the matrix inverse problem $A X=B$. Linear Algebra Appl., 289:131-134, 1999.

[9] J. Groß. Nonnegative-define and positive solutions to the matrix equation $A X A^{*}=$ B-revisited. Linear Algebra Appl., 321:123-129, 2000.

[10] J.C. Hou and J.L. Cui. Theory of Linear Mapping on Operator Algebras (in Chinese). Science Press, Beijing, 2002.

[11] I. Kaplansky. Modules over operator algebras. Amer. J. Math., 75:839-858, 1953. 
[12] C.G. Khatri and S.K. Mitra. Hermitian and nonnegative definite solutions of linear matrix equations. SIAM J. Appl. Math., 31:579-585, 1976.

[13] E.C. Lance. Hilbert $C^{*}$-modules $-A$ Toolkit for Operator Algebraists. Cambridge University Press, 1995.

[14] G.R. Wang. Generalized Inverse: Theory and Computations. Science Press, 2004.

[15] Q.W. Wang and C.Z. Dong. Positive solutions to a system of adjointable operator equations over the Hilbert $C^{*}$-modules. Linear Algebra Appl., 433:1481-1489, 2010.

[16] Q.W. Wang and C.Z. Dong. The general solution to a system of adjointable operator equations over the Hilbert $C^{*}$-modules. Oper. Matrices, 5(2):333-350, 2011.

[17] Q.W. Wang, J.W. Woude, and H. Chang. A system of real quaternion matric equations with applications. Linear Algebra Appl., 431:2291-2303, 2009.

[18] Q.W. Wang and Z.C. Wu. Common Hermitian solutions to some operator equations on Hilbert $C^{*}$-modules. Linear Algebra Appl., 432:3159-3171, 2010.

[19] Q. Wang and C. Yang. The Re-nonnegative definite solutions of the matrix equation $A X B=C$. Comment. Math. Univ. Carolinae, 39:7-13, 1998.

[20] Q.W. Wang and F. Zhang. The reflexive re-nonnegative definite solution to a quaternion matrix equation. Electron. J. Linear Algebra, 17:88-101, 2008.

[21] N.E. Wegge-Olsen. K-Theory and $C^{*}$-Algebras - A Friendly Approach. Oxford University Press, Oxford, 1993.

[22] L. Wu. The Re-positive definite solutions to the matrix inverse problem $A X=B$. Linear Algebra Appl., 236:141-151, 1992.

[23] L. Wu and B. Cain. The Re-nonnegative definite solutions to the matrix inverse problem $A X=B$. Linear Algebra Appl., 174:137-146, 1996.

[24] Q. Xu. Common Hermitian and positive solutions to the adjointable operator equations $A X=$ $C, X B=D$. Linear Algebra Appl., 429:1-11, 2008.

[25] X. Zhang. Hermitian nonnegative-definite and positive-define solutions of the matrix equation $A X B=C$. Appl. Math. E-Notes., 4:40-47, 2004.

[26] X. Zhang. The general common nonnegative-definite and positive definite solutions to the matrix equations $A X A^{*}=B B^{*}$ and $C X C^{*}=D D^{*}$. Appl. Math. Lett., 17:543-547, 2004. 